\title{
VIOLENCIA EN EL NOVIAZGO ADOLESCENTE
}

\author{
Yvonne Escoto Sainz, Marcela González Castro, Andrea Muñoz Sandoval y \\ Yannel Salomon Quintana ${ }^{1}$
}

\section{Resumen}

La presente investigación con diseño no experimental y de carácter descriptivo, determina si existe la violencia en cualquiera de sus manifestaciones en el noviazgo adolescente actual.

Se les aplicó un cuestionario a veinte mujeres adolescentes entre 15 y 18 años de edad, donde además se revelan los valores que no se encuentran involucrados en sus relaciones de pareja. Surgieron similitudes entre ellas obteniendo así que la mayor parte sufre de violencia tipo psicológica y, por otro lado, una minoría recibe abuso de tipo físico y sexual. Además se dejo ver que el respeto no es un valor que caracterice su relación de pareja. Son datos que nos van introduciendo al nuevo estilo de noviazgo que entre adolescentes se lleva, en el cual influyen muchos factores como familiares, ambientales, culturales, etc.; que, no importa donde ni cuando, siempre tendrán consecuencias que se reflejan en la calidad de las relaciones interpersonales.

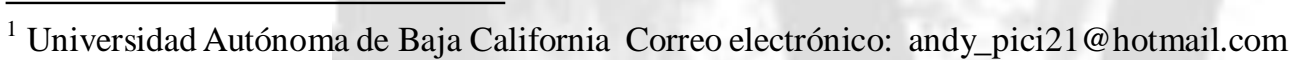




\section{Introducción}

Hoy en día, muchos adolescentes crean grandes expectativas alrededor del noviazgo, como lo son el amor, el cuidado, el apoyo y la compresión; por lo que a los jóvenes que viven relaciones violentas se les dificulta darse cuenta de dicha situación, y puede llegar a un punto en el que el noviazgo se convierta en un tormento.

No se conoce a ciencia cierta, cuales son los factores que influyen en la violencia; aunque algunas encuestas demuestran que puede ser la cultura, los roles que se le atribuyen a cada género, la familia, las amistades y los medios de comunicación.

Según 48 encuestas realizadas en todo el mundo, entre 10 y 69 por ciento de las mujeres, indicó haber sido objeto de agresiones físicas por parte de una pareja masculina en algún momento de su vida. (García, 2005).

En un estudio realizado por Gender (2004), sobre violencia en el noviazgo adolescente; el porcentaje de casos de violencia no sexual durante el cortejo es de 9 a un 65 por ciento, las amenazas y agresiones emocionales o verbales fueron incluidas en la definición.

De acuerdo con autoridades capitalinas, tan solo en el Distrito Federal; 9 de cada 10 jóvenes, entre 12 y 29 años sufren agresiones durante esta etapa de su vida (CONAPO, 2000).

El maltrato entre las parejas adolescentes mexicanas, duplica al que sufren las estadounidenses de origen hispano, reveló una encuesta binacional difundida en las ciudades fronterizas de Tijuana, monterrey y san diego. (Salas, 2003).

La violencia en el noviazgo es una situación de carácter serio, que no respeta estratos sociales, culturas, ni rangos de edades. No sólo consiste en violencia física como lo son los golpes, pellizcos, empujones, entre otras cosas; sino también se presenta la violencia verbal que consiste en insultos, amenazas, etc. Además un tercer tipo de violencia conocida como psicológica que engloba desde humillaciones, hasta la disminución de la capacidad de una persona para tomar decisiones con respecto a su propia vida, por esta razón es considerada la más grave de todas.

Según Sánchez (2003), al acercarse a escuchar las vivencias en las y los jóvenes y sus noviazgos, los testimonios indican que la violencia en el noviazgo aparecía ante la mirada juvenil como algo natural o normal, lo que les permitió atribuir el enorme reto de incidir en su urgente y necesaria prevención. 
Lo anterior, nos lleva a preguntarnos lo siguiente: ¿Se da la violencia en el noviazgo adolescente actual? Y si existe, cuál es la que más se presenta.

\section{Planteamiento del problema}

¿Se da la violencia en el noviazgo adolescente actual?

\section{Objetivo General}

Determinar si se da la violencia en el noviazgo adolescente actual.

\section{Objetivos Específicos}

Analizar que tipo de violencia existe en el noviazgo adolescente actual. Identificar los valores que se no presentan en un noviazgo adolescente actual.

\section{Justificación}

El tema de esta investigación, no es un tema por el cual se ha demostrado gran interés; sin embargo, es muy importante ya que se encuentra estrechamente ligado al bienestar de los jóvenes, tanto física como psicológicamente.

Con el paso del tiempo, los noviazgos han dado un cambio radical, es decir, los jóvenes ya no tienen las mismas expectativas con respecto al trato, valores, entre otras cosas, a las que tenían años atrás; la manera en que funcionan los noviazgos ha dado un gran giro.

Por lo antes mencionado, se considera que es muy importante y necesario que este tema sea explorado, porque el hecho de que haya dado un gran giro no significa que este sea para bien. Si no se conocen las características del noviazgo adolescente, esto podría representar un grave peligro por la violencia que se pudiera estar dando dentro 
del mismo, ya que dicha violencia no respeta nivel socieconómico, como tampoco escolar; es decir, no respeta nada.

Son estas las razones por las cuales se considera importante el seguimiento de esta investigación, ya que se espera que los datos obtenidos a partir de la misma, sean significativos y de esta manera poder situar la posible problemática real.

\section{Contexto de la investigación}

La investigación fue llevada a cabo en dos planteles educativos de Tijuana, Baja California, México. El primero de ellos fue la Facultad de Medicina de la Universidad Autónoma de Baja California, en la cual se encuentran las alumnas de la licenciatura en psicología que formaron parte la muestra. El segundo plantel educativo fue la Preparatoria Federal Lázaro Cárdenas, en donde también se encuentran alumnas que conformaron la muestra.

Variables

\begin{tabular}{|c|l|l|}
\hline Variable Independiente & Noviazgo & $\begin{array}{l}\text { Período de tiempo que } \\
\text { transcurre mientras dos } \\
\text { personas mantienen una } \\
\text { relación amorosa con o sin } \\
\text { intención de casarse. }\end{array}$ \\
\hline Variable Dependiente & Violencia & $\begin{array}{l}\text { Comportamiento deliberado } \\
\text { que resulta en daños físicos } \\
\text { psicológicos a otros seres } \\
\text { humanos. }\end{array}$ \\
\hline
\end{tabular}




\section{CAPÍTULO I}

\section{ADOLESCENCIA}

Reconocer la adolescencia como una etapa crucial del desarrollo humano, permite abandonar la imprecisión que involucró considerarla como una transición. Desde la perspectiva de transición los únicos hechos claramente reconocibles son el abandono de la niñez por las transformaciones biológicas que se presentan y el ingreso a la edad adulta, reflejado en los cambios de la situación social.

\footnotetext{
El desarrollo adolescente es un proceso de cambios y transformaciones, que permite un enriquecimiento personal y progresivo en una delicada interacción con los entes sociales del entorno; su valoración tiene como referente no solo la biografía del individuo, sino también la historia y el presente de su sociedad. (Krauskopf, 2000)
}

En la adolescencia se replantea la definición personal y social del ser humano a través de una segunda individuación que moviliza procesos de exploración, diferenciación del medio familiar, búsqueda de pertenencia y sentido de vida. Tal situación se hace crítica en los tiempos actuales, pues los y las adolescentes son los portadores de los cambios culturales.

Los adolescentes demandan, para el medio adulto, una reorganización de esquemas psicosociales que incluyen el establecimiento de nuevos modelos de autoridad e innovadoras metas de desarrollo. Sin las adecuadas condiciones, las nuevas interacciones personales y sociales pueden favorecer la emergencia de grandes riesgos y daños. (Krauskopf, 2000)

El desarrollo humano tiene por meta el enriquecimiento personal y social progresivo que avanza en la adolescencia mediante la actualización de capacidades que 
permitan la convivencia social positiva, rescatando las necesidades personales y el progreso colectivo en un ajuste e integración transformadores. La adolescencia es el periodo en que se produce con mayor intensidad de interacción entre las tendencias individuales, las adquisiciones psicosociales, las metas socialmente disponibles, las fortalezas y desventajas del entorno.

\section{Fasesdelperiodoadolescente}

Según Gómez (2001), en el funcionamiento psicológico, las dimensiones más destacadas del desarrollo adolescente se dan en el plano intelectual, sexual, social y en la elaboración de la identidad. Con la finalidad de facilitar la sistematización de sus características, se analizará la evolución que presentan a través de tres fases. Cabe aclarar, que no se trata de secuencias rígidas, pues las aceleraciones y desaceleraciones de los procesos dependen, a lo menos, de las diferentes subculturas, la situación socioeconómica, los recursos personales y tendencias previas, los niveles alcanzados de salud mental y desarrollo biológico, las interacciones con el entorno, y entre estas, las relaciones de genero y las relaciones intergeneracionales.

"Habitualmente las fases del periodo adolescente han sido denominadas Fase puberal, Adolescencia media y Adolescencia tardía. Preferimos identificar esta última como Fase Final del periodo adolescente”. (Gómez, 2001)

\footnotetext{
La fase puberal es la que se da entre los 10 y 14 años, la preocupación psicológica gira básicamente alrededor de lo físico y lo emocional. Se produce una reestructuración de la imagen corporal, se vive un ajuste a los cambios corporales emergentes, el ánimo es fluctuante; hay una fuerte autoconciencia de las necesidades y deseos de comprensión y apoyo por parte de los mayores. (Gómez, 2001)
}

Aún cuando las figuras parentales dejan de ser la fuente casi exclusiva de fomento de la autoestima, se hace imprescindible tener la posibilidad de compartir los problemas con los padres; las amistades también se tornan cruciales. Los grupos tienden 
a ser del mismo sexo, facilitando el fortalecimiento de identidades y roles antes de entrar a la interacción heterosexual.

Según Aberasturi (2000), tradicionalmente el comienzo de la pubertad marca la aparición del discurso en que los mayores enfatizan los riesgos y las perspectivas morales. La autonomía en la toma de decisiones no es fomentada. Culturalmente, la emergencia de indicios de desarrollo sexual tiende a ser enfrentada por los adultos de modo opuesto para cada sexo; control y vigilancia para las muchachas, estimulo y libertad para los varones.

De acuerdo con Gómez (2001), la adolescencia media se da aproximadamente entre los 14 y 16 años, las preocupaciones psicológicas giran prioritariamente en torno a la afirmación personal-social y afloran vivencias del amor. "La búsqueda de canalización de los emergentes impulsos sexuales, la exploración de las capacidades sociales, y el apoyo en la aceptación peor el grupo de pares dinamizan la afirmación personal y social en la adolescencia."

La familia ha dejado de ser el espacio privilegiado para confirmar las habilidades y autoestima adolescente, lo que genera para las figuras parentales el difícil desafío de lograr la capacidad de mantener y expresar, en estas nuevas condiciones, la aceptación de sus hijos adolescentes, lo que es siempre fundamental para su desarrollo.

La sexualidad adolescente debe ser vivida fuera de la familia y los nuevos roles son ensayados y comprobados en grupos de pares y ámbitos de la sociedad mas amplia. Esto conforma nuevas condiciones para el desarrollo social que contribuyen a la diferenciación del grupo familiar y a la autonomía. (Gómez, 2001).

Es importante reconocer que para las y los adolescentes, el amor, el goce y la amistad son preocupaciones muy valiosas que tienden a suprimirse por los adultos 
cuando enfocan su sexualidad desde el ángulo exclusivo de la moral o la salud reproductiva. No es infrecuente que para las muchachas los novios sean la figura mayor de confianza para compartir afectos y preocupaciones.

Las relaciones sentimentales sirven a muchachos y a muchachas como oportunidades para ampliar experiencias e intereses y enriquecer la identidad y no son, generalmente, noviazgos orientados a la unión conyugal, salvo en zonas rurales.

Según Gómez (2001), en la última fase de la adolescencia se comienza a evolucionar de un proyecto de vida complementario con el proyecto familiar a una forma de enfrentamiento personal y social propia que deberá ir poniendo a prueba en la práctica concreta y aportara a la consolidación de la identidad y roles.

No se trata de la elaboración de un proyecto planificado de principio a fin, como podía esperarse tradicionalmente, sino del compromiso con pasos y experiencias dadas es su presente, que constituyan vías flexibles hacia los roles y metas de acuerdo con al incertidumbre de los tiempos, las figuras parentales enfrentan el duelo que provoca el desprendimiento físico del medio familiar por el adolescente.

Las capacidades de autocuidado y cuidado mutuo tienen la posibilidad de despegarse eficazmente, en la medida que los y las adolescentes hayan contado con la asesoría y atribuciones requeridas. Las parejas dejan de cumplir el rol de exploración y descubrimiento de mundos emocionales y sexuales, para introducir como vivencia central, la apertura en la intimidad que emerge entre las personas con identidades mas diferenciadas que se enriquecen con el acompañamiento afectivo y el establecimiento de vínculos profundos. 


\section{Consideracionesfinales}

Según Martínez (2003), el periodo adolescente constituye una etapa privilegiada en la cual se producen procesos claves del desarrollo, contando por primera vez, con la propia capacidad para conducir el proceso, completar vacíos y reenfocar situaciones tanto de la niñez, como su presente.

Los cambios físicos hormonales, sexuales, emocionales e intelectuales desencadenan necesidades, riesgos, respuestas individuales, sociales e interactivas. La elaboración de la identidad pone a prueba las fortalezas y debilidades propias y del entorno.

\footnotetext{
Con la adolescencia, personas nacen para la sociedad más amplia y por lo tanto se inicia un desprendimiento del sistema familiar que lleva a una resignificación de las relaciones. Se desencadenan procesos que van concretando las bases para la construcción de los roles y perspectivas de la vida en el contexto de las demandas, recursos y limitaciones que ofrecen las sociedades en sus entornos específicos y en un momento histórico político dado. (Martínez, 2003).
}

Un desafío importante es hacer coincidir los elementos identitarios asumidos con acciones que promuevan el desarrollo y con la adquisición de instrumentos que favorezcan la consolidación de roles satisfactorio

\section{CAPÍTULO II}

\section{GÉNERO}

Las mujeres y los hombres tienen diferencias de acuerdo al sexo, es decir, diferencias de carácter biológico. Los seres humanos somos seres sexuados, tenemos sexo definido. Nuestro cuerpo sexuado, viene a constituir la manera de relacionarnos con otros seres, por que es mediante esta diferencia que se va a d dar nuestra forma de comportarnos y a los valores que vamos a tener hombres y mujeres en la sociedad. De hecho, el 
nacimiento de una niña o un niño implica expectativas distintas sobre cual debe de ser el comportamiento de una a otra.

"El género se refiere al aprendizaje que vamos adquiriendo mujeres y hombres, de acuerdo a la cultura y sociedad en que vivimos, asumiendo así roles o patrones de comportamiento, de acuerdo al sexo que tenemos." (García, 2001)

Las distintas sociedades, han atribuido significados diferentes a cada género, estableciendo una serie de características para cada uno, lo que se ha denominado, identidad de género. Esto significa que se aprende a ser mujer y hombre, y por lo tanto lo femenino y lo masculino son características socioculturales y no naturales.

\section{Identidaddegénero}

Según Moya (2000), “La identidad de género es la percepción interior de la persona como hombre o mujer."

La identidad es la imagen que las personas logran construir de sí mismas, la forma como se auto-perciben, su auto-imagen y la forma en que interactúan en sociedad a partir de esta auto-percepción.

Según Barrio (1992) "La identidad tiene algunas características fundamentales, está referida a un contexto social y cultural, se afirma en la relación interpersonal, y es producto tanto de la sociedad como de la acción de las propias personas."

La identidad de género cobra sentido, en la medida en que mujeres y hombres interiorizamos atributos, formas de ser y actuar acorde a lo socialmente definido, como masculino y femenino. Es decir, asumimos y representamos patrones de conducta diferentes de acuerdo a si somos mujeres u hombres. 


\begin{abstract}
Conocer el cómo se nos enseña a ser mujer y a ser hombre, los roles de género y sus prescripciones, la socialización de género y sus respectivos resultados para cada sexo, nos ayudan a entender cómo se va preparando el terreno para que un muchacho maltrate a su novia y para que este permanezca en una relación violenta. Un muchacho que quiera cumplir cabalmente con el rol de género masculino tradicional será propenso a la violencia; aparentar la actividad sexual, ser quien tome las decisiones en la relación de pareja, dominar y controlar las actividades de ella, probar constantemente que "él es el hombre" a través de actos agresivos y dureza por temor al estigma de la homosexualidad, esperar que la novia renuncie a sus intereses o a otras relaciones y de la máxima prioridad a su relación con él (Troncoso y Tenorio A., 1999).
\end{abstract}

\title{
$\underline{\text { Roles }}$
}

Los roles de genero en cada cultura son diferentes, en México es común que la familia sea dirigida por el hombre, por el clásico estereotipo de que los hombres son fuertes, inteligentes, protectores y las mujeres sumisas, y el hombre es quien las debe proteger. Esto es un claro ejemplo de querer ser el macho mexicano, es muy común que los hombres por sus inseguridades traten de aparentar algo que no son y al poder dominar a la mujer se sienten seguros de sí mismos, cuando todo es una realidad disfrazada de temor.

Los hombres desde pequeños aprenden a resolver las diferencias por medio de la violencia, son violentos cuando tienen algún problema en la calle, además los juegos contienen violencia al igual que la televisión y otros medios que muestran la violencia social en la que actualmente vivimos.

\footnotetext{
"Una muchacha puede ceder a la presión de grupo e intentar cumplir con las prescripciones del rol de genero femenino tradicional, creer que puede cambiar al hombre que ama; sentirse culpable por los problemas y responsable del funcionamiento de la relación, pensar que jamás encontrará a otro novio, lo que la pondrá en riesgo de involucrarse en relaciones potencial o abiertamente violentas." (Troncoso y Tenorio, 1999).
}

Con respecto al rol de la mujer anteriormente citado de cierta manera es lo que se nos ha impuesto a nuestra cultura que tiene que aguantar todo lo que el hombre le hace, como si fuera su destino. Como se había mencionado anteriormente, aquí también 
se presenta un problema de autoestima en la mujer cuando se deja agredir por la pareja; no se deja valer como debe hacerlo, por miedo a quedarse sola o por que simplemente piensa que ella es menos que él.

\footnotetext{
"La identificación es la reproducción de roles o patrones de comportamiento en una sociedad; de acuerdo con los significados y valoraciones de esa sociedad y cultura particular que se concretan por medio de una red de relaciones y de muy diversa manera." (Suárez, 2000).
}

Las niñas y los niños aprenden los modelos femenino y masculino socialmente aceptados, a partir de los cuales, construyen su auto-percepción y valoración de las otras personas con las que se relaciona.

\section{CAPÍTULO III.}

\section{NOVIAZGO}

Se trata de una situación de tránsito en las relaciones entre un hombre y una mujer antes de casarse.

El noviazgo en su forma actual se caracteriza por una mayor libertad e independencia de criterio, frente a lo que era práctica habitual en otras épocas, a la hora de elegir pareja. A la vez la relación hombre-mujer en el matrimonio se va alejando de los patrones tradicionales. Puede ser una excelente escuela de formación de la voluntad, que combate el egoísmo, fomenta la generosidad y el respeto, estimula la reflexión y el sentido de responsabilidad.

"El noviazgo debe ser una ocasión de ahondar en el afecto y en el conocimiento mutuo. Y, como toda escuela de amor, ha de estar inspirada no en el afán de posesión sino por 
el espíritu de entrega, de comprensión, de respeto, de delicadeza”. (Josemaría Escrivá de Balaguer)

\footnotetext{
Según Rivas (2000), "El noviazgo va más allá de la mera relación de amistad. Se añade una atracción hacia el otro de un orden especial, inmaterial, espiritual, que se quiere fomentar, enriquecer, cultivar, en orden a resolver dos cuestiones que entonces se plantean: una, si es posible la continuidad de esa relación que ha surgido; y otra, si puede convertirse en matrimonial. Es decir, el noviazgo se vive en relación a un posible o incluso probable matrimonio, del cual obtiene sus propias características. Es decir, de cómo se entienda el matrimonio, depende lo que se entienda por noviazgo".
}

\section{Valoresenelnoviazgo}

En algún momento de nuestra vida nos descubrimos ilusionados y cautivados por una persona en especial. Podemos decir que nada se compara con la presencia de "ese alguien" y nuestro cariño se fortalece en la medida que encontramos correspondencia a las atenciones y detalles que mutuamente se procuran en pareja.

De acuerdo con Franco (2001), pocas son las parejas que pudieran servir de modelo para ilustrar como debe vivirse el noviazgo. Los más nobles sentimientos parecen estar sometidos a unos cuantos encuentros casuales, al placer sensual, a rendir juicio y voluntad a las exigencias de la otra persona. Tal vez la modernidad propone una vida sin compromisos, donde al final de cuentas lo importante es pasar un buen rato.

\section{Respeto}

Siempre se habla de respetar ideas, opiniones, gustos, hábitos y costumbres, pero eso no significa estar de acuerdo con todo, o permanecer indiferentes. Muchos de estos aspectos pueden no parecernos e incluso disgustarnos y lo correcto será ayudarse mutuamente a corregirlos.

Según Rivas (2000), cuando existe un interés y cariño auténticos, siempre se buscará el bien de la otra persona. El respeto en pareja también debe vivirse en las 
manifestaciones de cariño como lo son las caricias, los abrazos, los besos, la toma de decisiones, e incluso las palabras.

\section{Sana Diversión}

De acuerdo con Franco (2001), “uno de los más grandes peligros que existen en los noviazgos, es la búsqueda constante de nuevas diversiones. Lo más difícil es encontrar actividades que nos permitan vivir los valores y conducirnos con respeto". La ociosidad y la falta de prudencia en pareja constituyen la causa de todos los desaciertos que se cometen. Por eso ayuda mucho el asistir a lugares públicos con adecuada iluminación; realizar actividades en conjunto, platicar en casa o ver películas a puertas abiertas, además de cerciorarse que alguna otra persona se encuentra en el lugar; tener alguna afición: deportes, música, pintura, etc.

Es importante recordar que los lugares y actividades donde circula el alcohol y drogas, disminuyen nuestra capacidad de autodominio; por lo tanto, no deben considerarse como la mejor opción para divertirse.

\section{Autoestima}

“Cuando la autoestima está bien fundamentada, se aprende a aceptar los consejos y críticas para mejorar nuestra persona: modales, vocabulario, forma de vestir, disposición al trabajo o al estudio, relación familiar, etc.” (Rivas, 2000). Pero también nos ayuda a defender nuestros principios morales, rechazar la manipulación de nuestras ideas y sentimientos para no ceder a los caprichos de otra persona, ya sea cambiando nuestra conducta, creencias, amistades o acceder a tener relaciones por miedo a perder a la pareja.

\section{Compromiso}

De acuerdo con Rivas (2000), en todas las relaciones humanas hace falta comportarse con madurez y seriedad; el noviazgo no es una relación de segunda categoría por ser 
considerado como algo informal. Esto significa evitar jugar con los sentimientos de la otra persona, engañarla o buscar su compañía para cuando no haya algo mejor que hacer.

De alguna manera, al vivir lo mejor posible un noviazgo las personas se preparan para adquirir mayores compromisos, como puede ser el matrimonio. Lo cierto es que la falta de compromiso en cualquier actividad o relación, termina por llevarnos a la superficialidad, la apatía y la trampa.

Lo anterior no quiere decir que una pareja debe permanecer "atada" indefinidamente. Se trata de poner en orden nuestros intereses, planes, actividades y sentimientos, para encontrar el momento justo y la persona adecuada para emprender una relación.

Devolver el encanto al noviazgo es recuperar el respeto y la dignidad de hombres y mujeres, convirtiéndose en personas con la capacidad de formar una familia que vive y transmite valores humanos a la sociedad.

\section{Fidelidad}

Según Monge (2000), en el noviazgo existe un compromiso de fidelidad pero también es cierto que no es lo mismo ahora que antes. En todo caso, en estos tiempos lo que se debe pedir es lealtad para evitar que exista un doble juego durante mucho tiempo; es decir, un engaño.

Con lo anterior; se quiere dar referencia, al hecho de que debe de existir una libertad para cambiar de pareja; cuando alguno de los que conforman la misma lo desee pero sin dejar de lado el respeto y la honestidad por la persona actual.

\section{Celosenelnoviazgo}

Según García (2002), los celos son emociones intensas que surgen cuando se experimenta un deseo exagerado de poseer de forma exclusiva a la persona amada, a un 
amigo o a los hijos. Los psiquiatras afirman que los celos son un estado antecesor de la psicosis, pueden convertirse en delirio y perturbar el sentido de la realidad.

De acuerdo con Casavantes (2000), los celos enfermizos llegan a provocar, en infinidad de casos, violencia física y psicológica continuas, e incluso que un miembro de la pareja acabe con la vida del otro. Los ataques de celos son el motivo más frecuente de homicidios conyugales y un factor determinante en casi el $20 \%$ de todas las agresiones violentas en los países desarrollados.

Freud estaba convencido de que los celos tienen sus raíces en experiencias infantiles que ya todos vivimos, para él los celos patean el amor propio y el orgullo. En su interpretación encuentra en los celos causas biológicas, psicológicas y sociales. Menciona que hay celos que tienen como origen la química del sistema nervioso, los celos llamados delirantes se originan en desordenes de las sustancias neurotransmisoras. La posesividad exagerada es una expresión de inmadurez amorosa que provoca finalmente la sofocación de la persona amada y finalmente la ruptura del vínculo. (Godos, 2001).

\section{CAPÍTULO IV}

\section{VIOLENCIA EN EL NOVIAZGO}

Según el IMJ (2004), La violencia es un problema social de gran impacto que atraviesa fronteras raciones, de edad, religiosas, educativas y socioeconómicas, es un fenómeno que lesiona los derechos de los seres humanos y muestra de forma nítida la opresión de género.

La violencia en el noviazgo ocurre en una relación amorosa en que una de las personas abusa física, emocional o sexualmente para dominar y mantener el control sobre la otra.

De acuerdo con Pacay (2003), el noviazgo en los adolescentes se encuentra basado en una conquista de tipo emocional, en la que cada uno de los implicados trae 
sus objetivos y nunca se llegan a fusionar. En este tipo de relaciones lo único que prevalece es la atracción física, el interés, la duda y la curiosidad.

\footnotetext{
En nuestras relaciones el amor y la violencia han transitado inexplicablemente juntos los distintos senderos, pero con grandes consecuencias y repercusiones, en particular hacia las mujeres. Destrabar esta relación implica analizar y pensar en el amor de distinta forma, en la cual la violencia no tenga cabida, pero para llegar a eso es necesario antes describir y visibilizar nuestras relaciones amorosas; se necesita darle el valor de anormal o antinatural, a lo que en un sinfín de ocasiones nos han dicho que es lo normal. (Baños, 2000).
}

Continuando con Baños (2000), el noviazgo se muestra como un terreno fértil para el germen de la violencia, debido a que en éstas etapas las y los jóvenes inician su experiencia en las relaciones de pareja aceptadas socialmente, en el cual se despliegan de manera significativas los roles y estereotipos aprendidos, con el propósito de cumplir con una de las principales expectativas sociales: el matrimonio.

La violencia en el noviazgo, no es fácil de detectar y puede tener como repercusiones el fracaso escolar y en el peor de los casos depresión, aislamiento e intento de suicidio.

Según Magally (2002), la violencia en el noviazgo pasa desapercibida también porque el maltrato se asocia a las parejas casadas y con hijos, no se reconoce, principalmente por la desvalorización de las relaciones amorosas entre adolescentes; ya que son consideradas como cosas de la edad.

Lo anterior también se debe a que la conducta violenta no es percibida por las víctimas; mujeres en su mayoría, así como tampoco la perciben quienes la ejercen; hombres por lo general, y esto es porque es confundida con una expresión de interés y de amor.

\section{Tiposdeviolencia}


En el noviazgo la violencia puede adoptar múltiples rostros como lo son los gritos, amenazas, burlas, empujones, puntapiés, intimidaciones, castigos, asecho, sometimiento, bofetadas, críticas negativas, puñetazos, agresiones con objetos, agresiones con armas, etc. (Magally 2002).

\begin{abstract}
Abuso físico
Este tipo de abuso se ejerce mediante la fuerza física en forma de golpes, empujones, patadas y lesiones provocadas con diversos objetos o armas. Puede ser cotidiana o cíclicamente, y se ven combinados momentos de violencia física con periodos de tranquilidad.

Según Favieres (2001), en ocasiones suele terminar en suicidio u homicidio. El maltrato físico se detecta por la presencia de magulladuras, heridas, quemaduras, moretones, fracturas, dislocaciones, cortes, pinchazos, lesiones internas, asfixia o ahogamientos.
\end{abstract}

\begin{abstract}
Abuso sexual
Este tipo de abuso es difícil de demostrar a menos que vaya acompañado por lesiones físicas. Se produce cuando la pareja fuerza a la mujer a mantener relaciones sexuales o le obliga a realizar conductas sexuales en contra de su voluntad.

De acuerdo con Favieres (2001), los principales malos tratos sexuales son las violaciones vaginales, las violaciones anales y las violaciones bucales. También son frecuentes los tocamientos y las vejaciones, pudiendo llegar hasta la penetración anal y vaginal con la mano, puño u objetos.
\end{abstract}




\section{Abuso psicológico}

Los factores que influyen en el abuso psicológico son muy variados: emocionales, sociales, etc. La mujer se ve dominada por el varón, quien la humilla en la intimidad y públicamente, al igual que limita su libertad de movimiento, así como la libertad para relacionarse con los demás.

Algunos de este tipo de violencia son comentarios como los siguientes: "flaquita, ¿no crees que estás muy maquillada?", "sería bueno que dejaras de pasar tanto tiempo con tus amigos/as", "no me gusta como se te ve esa falda, porqué mejor no te pones un pantalón", “me gustaría que hicieras esto o aquello”. (Baños, 2000).

Muchas de las veces las jovencitas se encuentran conformes con lo anterior, pero mientras se va desarrollando la relación paulatinamente este tipo de violencia va cobrando mayor intensidad y con frecuencia reproduciendo círculos de violencia.

\footnotetext{
"Resulta complicado detectar este tipo de abuso, aunque se evidencia a largo plazo en las secuelas psicológicas. En este caso la violencia se ejerce mediante insultos, vejaciones, críticas, crueldad mental, gritos, desprecio, intolerancia, humillación en público, castigos o amenazas de abandono. Conduce sistemáticamente a la depresión y, en ocasiones, al suicidio". (Faviere, 2001).
}

\section{Gravedaddelabuso}

Favieres (2001), también menciona que la gravedad de estos abusos varía en virtud del grado de violencia ejercida sobre la mujer y normalmente se combinan varios tipos de abuso, ya que dentro del maltrato físico siempre hay un maltrato psicológico.

Evidentemente, el maltrato físico severo puede dejar secuelas muy graves, como la pérdida de audición, entre otras cosas; pero las secuelas psicológicas son las que más perduran, y es el tipo de violencia más difícil de detectar por que puede ser ejercida sutilmente.

Las jovencitas que son victimas del maltrato psicológico muchas de las veces van cambiando su comportamiento paulatinamente; es decir, van cediendo a todo lo que 
su pareja les pide y esto es algo muy grave debido a que su pareja se vuelve una parte importante en la toma de decisiones. (Favieres, 2001).

\section{Consecuencias}

Según Domínguez (2002), algunas de las consecuencias de la violencia son: depresión, aislamiento, fracaso escolar, bajo rendimiento laboral, etc. La violencia en el noviazgo puede ser el inicio de una vida en pareja y posteriormente en familia marcada por el maltrato.

\footnotetext{
La falta de educación acerca de este problema es especialmente grave para las adolescentes debido a la naturaleza cíclica de la violencia doméstica. Muchos niños que crecieron en hogares violentos recrean este ambiente en sus relaciones de pareja y las jovencitas que experimentaron maltrato de niñas por parte de sus padres tienden a involucrase al crecer con parejas igualmente violentas. (Arredondo, 2001)
}

Cabe señalar que estos signos de violencia se veían desde fuera como cosas de la edad, esto es, que en una relación de novios era común y válida la manifestación del maltrato, con el pretexto de que "ya llegarán a la edad adulta y se revitalizará la problemática". (Baños, 2000). 


\section{MÉTODO}

\section{Enfoque}

El enfoque de la investigación es de tipo cuantitativo; según Sampieri (2004), dicho enfoque consiste en cuantificar y aportar evidencia a una teoría que se tiene para explicar algo, la teoría se mantiene hasta que se refute o se alcance una mejor explicación.

\section{Hipótesis}

En la actualidad, en el noviazgo adolescente se presenta la violencia en sus diferentes manifestaciones.

\section{Diseño}

El diseño aplicado en esta investigación es no experimental; de acuerdo con Sampieri (2004), “este diseño podría definirse como la investigación que se realiza sin manipular deliberadamente variables". En esta investigación no se modifican las variables independientes, sólo se observan como se presentan en su contexto natural.

La investigación es de tipo descriptivo, ya que sólo se recolectarán datos sobre la violencia en el noviazgo adolescente, que es el fenómeno a investigar. Continuando con Sampieri (2004), "los estudios descriptivos buscan especificar las propiedades, las características y los perfiles importantes de personas, grupos, comunidades o cualquier otro fenómeno que se someta a un análisis”.

Este también es un diseño transversal, debido a que los datos solo serán recolectados una vez; de acuerdo con el citado autor, los diseños de investigación transversal recolectan datos en un solo momento. Su propósito es describir variables y analizar su incidencia e interrelación en un momento dado (o describir comunidades, eventos, fenómenos o contextos). 


\section{G-O}

$\mathbf{G}=$ Muestra (mujeres de 15 a 18 años con pareja).

$\mathbf{O}=$ La aplicación del instrumento solo se llevará a cabo una vez.

\section{Descripción de la muestra}

La muestra de esta investigación es una no probabilística, ya que los sujetos deben de ser mujeres adolescentes entre 15 y 18 años de edad, que tengan una relación de noviazgo en el momento de recolección de datos.

\section{Instrumento}

El objetivo del instrumento es determinar si se presenta la violencia en el noviazgo adolescente actual; consta de dos posibles respuestas que son: si y no, en el cual la respuesta "si" equivale a dos puntos y la respuesta "no" a uno. Para que sea posible obtener la puntuación final se tiene que llevar a cabo una sumatoria de todos los puntos obtenidos en los reactivos.

La máxima puntuación que puede ser obtenida en dicha herramienta es igual a cincuenta y ocho puntos y la mínima puntuación que se puede obtener es igual a veintinueve puntos. El cuestionario consta de veintinueve reactivos que se encuentran agrupados en tres diferentes categorías:

a) Reactivos 1-23: Violencia en el noviazgo Cap. 4

b) Reactivos 24: Roles Cap. 2

c) Reactivos 25-29: Valores-noviazgo Cap. 3

Por ultimo, la forma de aplicación del instrumento es muy sencilla, se le entrega al sujeto el cuestionario y se le pide que lo conteste. No tiene que ser aplicado en un 
lugar específico, el único requisito es que cumplan con las características necesarias

para la investigación (mencionadas en el punto \#2).

\section{Universidad Autónoma de Baja California Licenciatura en Psicología}

\section{Edad}

\section{Instrucciones.- Favor de seleccionar con una $X$ la respuesta que consideres adecuada.}

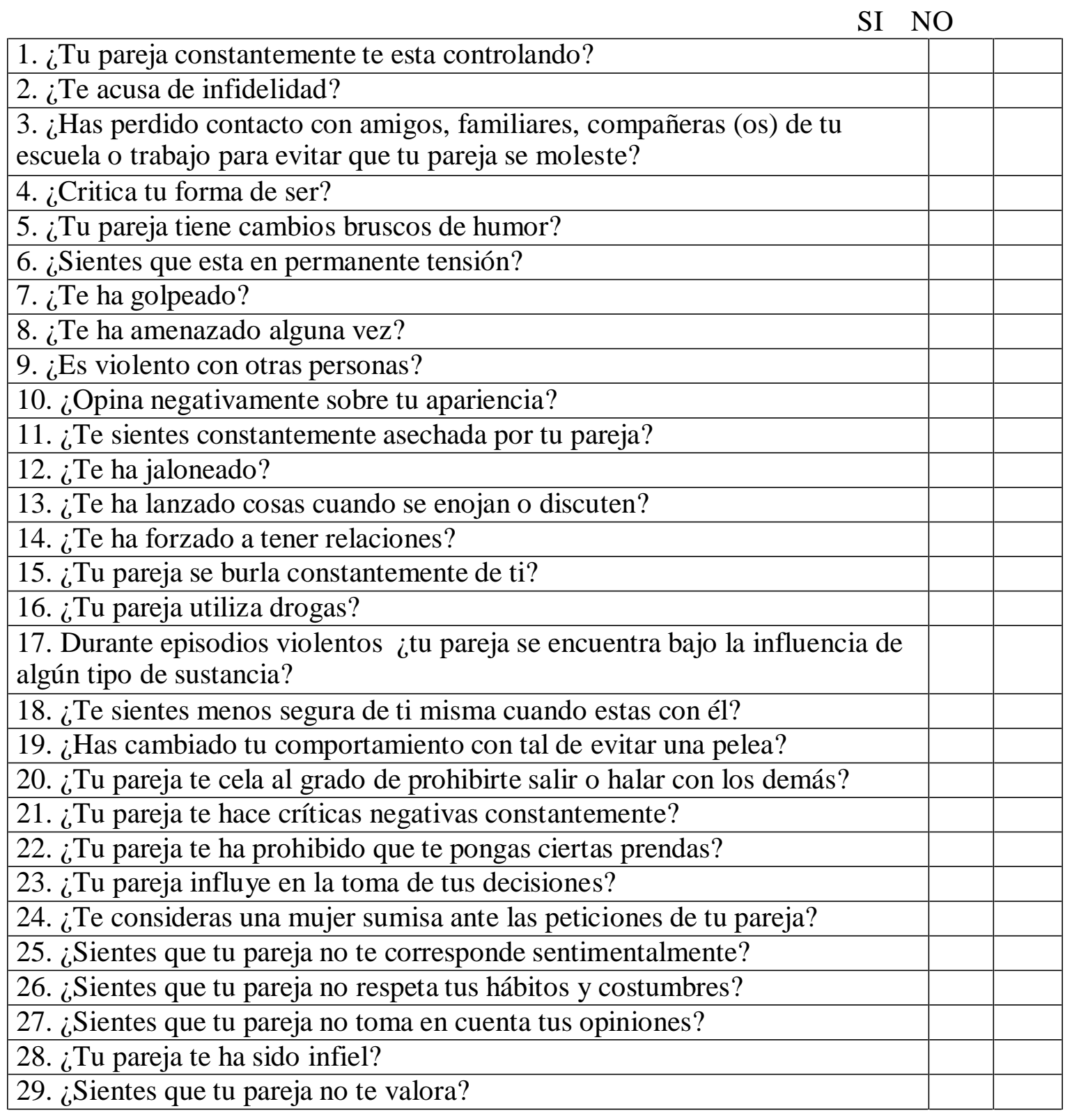




\section{Procedimiento}

Se asistió a la Universidad Autónoma de Baja California para llevar a cabo la aplicación del instrumento de la presente investigación a los primeros diez sujetos de 18 años de edad que corresponden al primer semestre de la carrera de psicología.

Se acudió a tres salones de clases, en donde se les explicó a los maestros que se está llevando a cabo una investigación, con el propósito de que permitieran realizar la aplicación de los cuestionarios; para de esta manera obtener los datos necesarios.

Por otra parte, para continuar con la recolección de datos se acudió a la Preparatoria Federal Lázaro Cárdenas en donde se logró reunir a veinte sujetos para obtener una muestra representativa cuyos resultados se apegaran a los objetivos de esta investigación.

En esta última institución educativa se llevó a cabo la medición de la violencia en el noviazgo adolescente cuando las jóvenes se encontraban fuera de los horarios de clases; se les explicó que se estaba efectuando una investigación en donde su participación era vital para la realización de la misma. 


\section{Resultados}

Tabla 1. Pérdida de contacto

\begin{tabular}{|c|c|}
\hline RESPUESTAS & FRECUENCIA \\
\hline SI & 18 \\
\hline NO & 12 \\
\hline TOTAL & 30 \\
\hline
\end{tabular}

\section{Grafica 3. Pérdida de contacto}

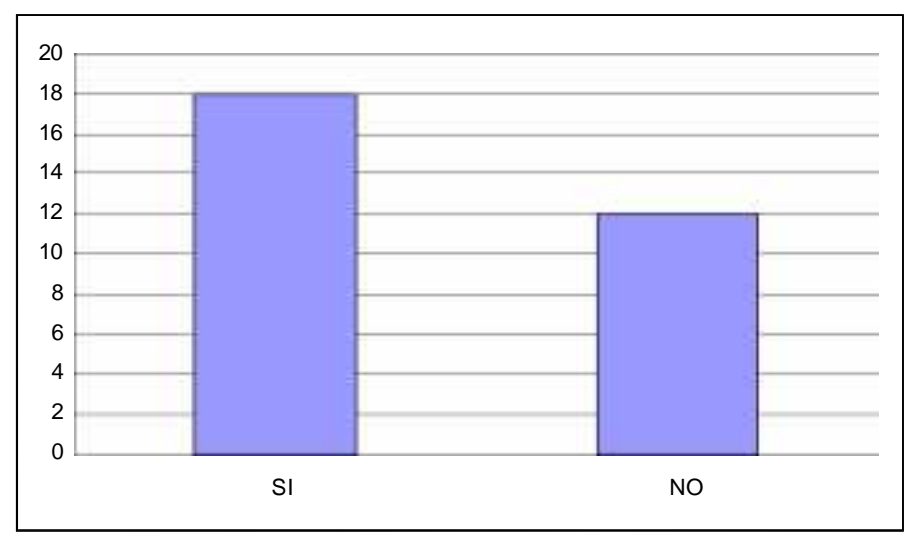

En esta grafica se puede observar que el $60 \%$ de las adolescentes han perdido contacto con amigos, familiares, compañeros de escuela o trabajo para evitar que su pareja se moleste; a diferencia del $40 \%$ restante que no lo ha perdido.

Tabla 1.1. Critica forma de ser

\begin{tabular}{|c|c|}
\hline RESPUESTAS & FRECUENCIA \\
\hline SI & 21 \\
\hline NO & 9 \\
\hline TOTAL & 30 \\
\hline
\end{tabular}

\section{Grafica 3.1. Critica forma de ser}

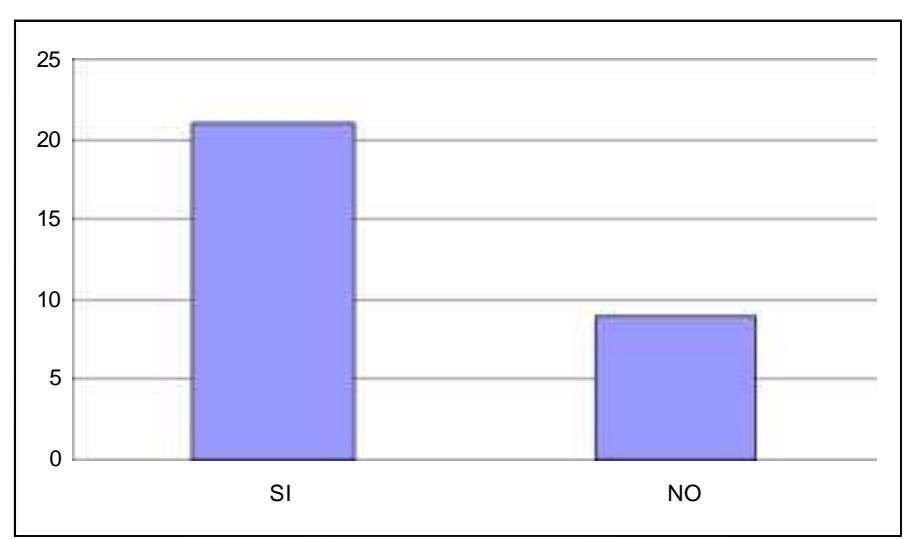

Esta grafica muestra que el $70 \%$ de las jovencitas encuestadas son criticadas en cuanto a su forma de ser por sus parejas; en cambio, el $30 \%$ de las mismas respondieron que sus novios no lo hacen.

\section{Tabla 1.2. Golpes}




\begin{tabular}{|c|c|}
\hline RESPUESTAS & FRECUENCIA \\
\hline SI & 5 \\
\hline NO & 25 \\
\hline TOTAL & 30 \\
\hline
\end{tabular}

\section{Grafica 3.2. Golpes}

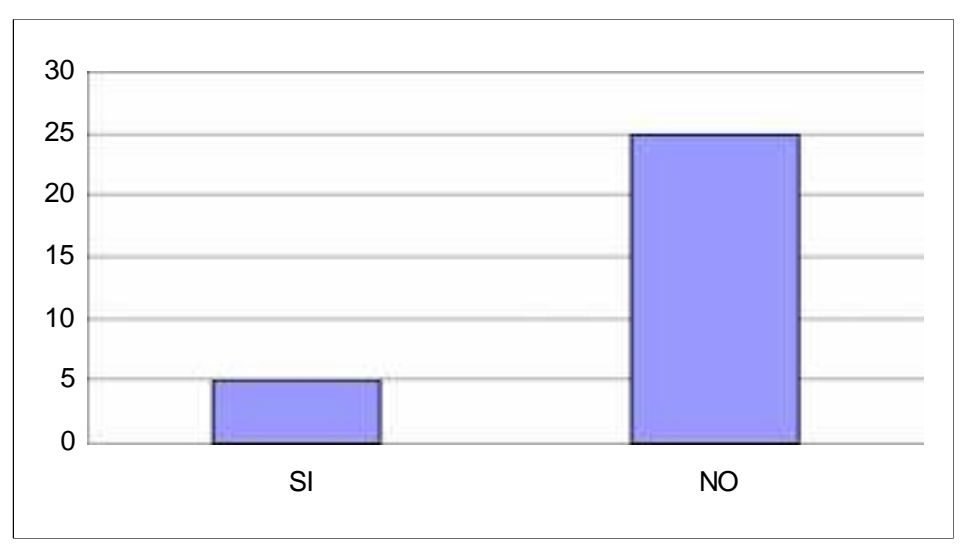

En esta grafica se puede observar que el $83.3 \%$ de las jovencitas encuestadas dijeron que sus novios no las han golpeado; en cambio, el $16.7 \%$ dijo que si habían recibido golpes por parte de sus novios.

Tabla 1.3. Relaciones

\begin{tabular}{|c|c|}
\hline RESPUESTAS & FRECUENCIA \\
\hline SI NO & 4 \\
\hline TOTAL & 26 \\
\hline & 30 \\
\hline
\end{tabular}

\section{Grafica 3.3. Relaciones}

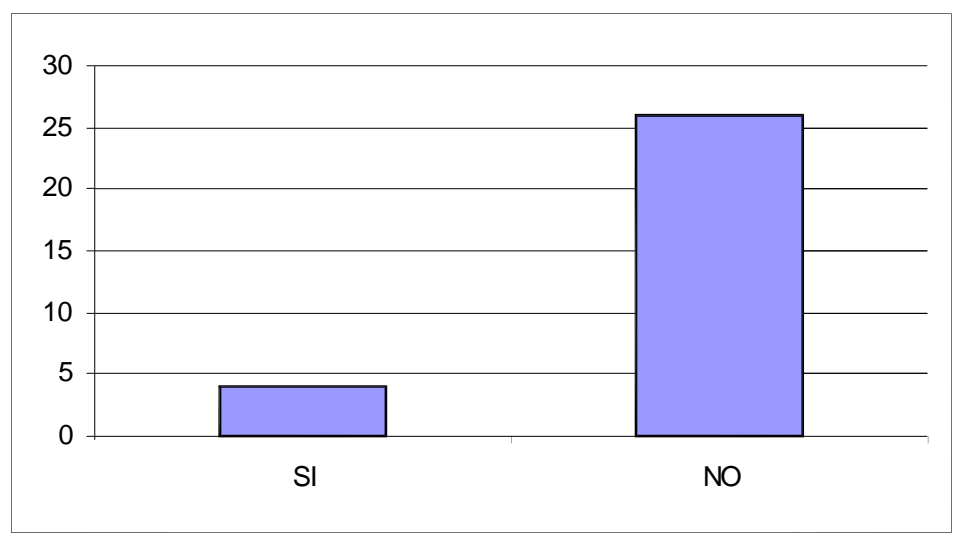

Se observa en esta grafica que el $86.7 \%$ dijo que no se les ha forzado a tener relaciones sexuales; en cambio, un $13.3 \%$ reveló que si han sido forzadas a tenerlas. 
Tabla 1.4. Burla constante

\begin{tabular}{|c|c|}
\hline RESPUESTAS & FRECUENCIA \\
\hline SI & 17 \\
\hline NO & 13 \\
\hline TOTAL & 30 \\
\hline
\end{tabular}

\section{Grafica 3.4. Burla constante}

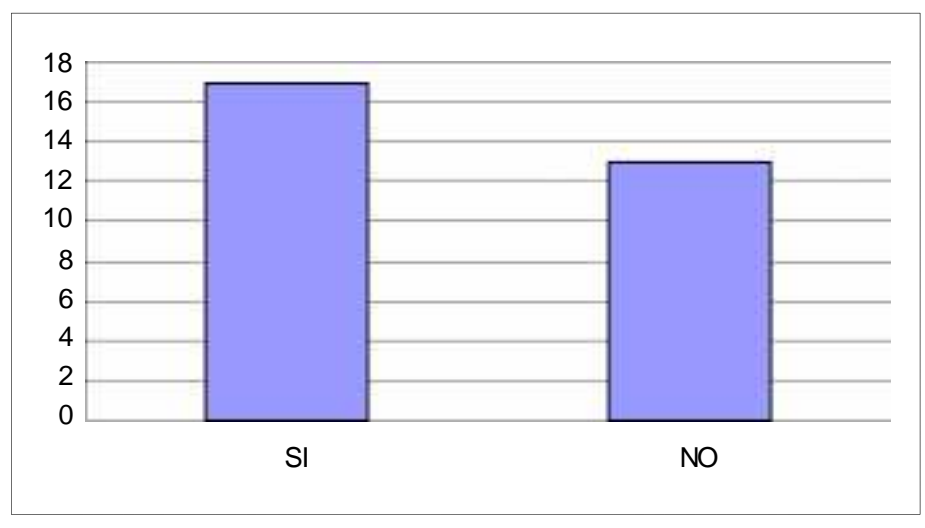

Esta grafica muestra que el $56.7 \%$ de las jovencitas dicen que sus parejas se burlan constantemente de ellas, mientras que el $43.3 \%$ afirma que no.

Tabla 1.5. Cambio de comportamiento

\begin{tabular}{|c|c|}
\hline RESPUESTAS & FRECUENCIA \\
\hline SI & 18 \\
\hline NO & 12 \\
\hline TOTAL & 30 \\
\hline
\end{tabular}

\section{Grafica 3.5. Cambio de comportamiento}

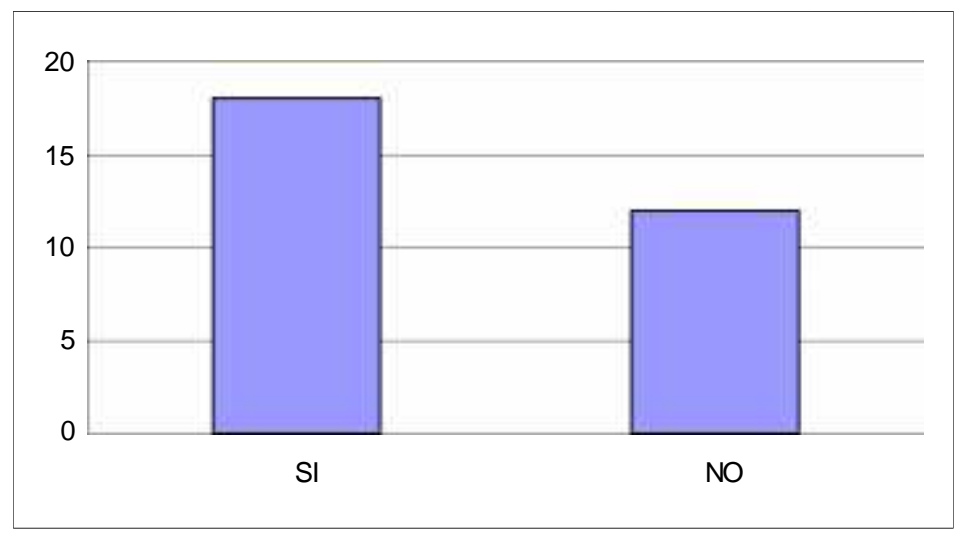

Se observa en esta grafica que el $60 \%$ de las adolescentes encuestadas han cambiado su comportamiento con tal de evitar una pelea con su pareja, a diferencia del $40 \%$ restante que no lo ha hecho. 
Tabla 1.6. Celos

\begin{tabular}{|c|c|}
\hline RESPUESTAS & FRECUENCIA \\
\hline SI NO & 20 \\
\hline TOTAL & 10 \\
\hline & 30 \\
\hline
\end{tabular}

\section{Grafica 3.6. Celos}

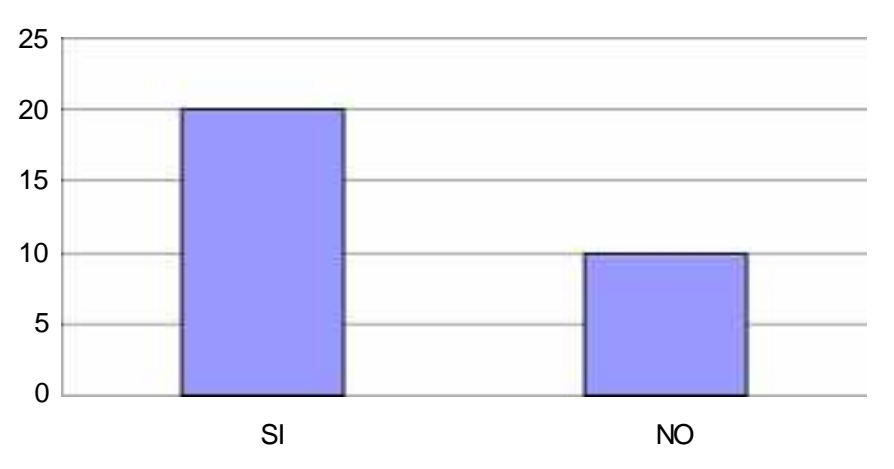

En esta grafica se observa que el $66.7 \%$ de las jovencitas han sido celadas por sus novios al punto de que éstos les han prohibido salir o hablar con los demás, mientras que el $33.33 \%$ de ellas respondieron que no se han encontrado en esta situación.

Tabla 1.7. Críticas negativas

\begin{tabular}{|c|c|}
\hline RESPUESTAS & FRECUENCIA \\
\hline SI & 18 \\
\hline NO & 12 \\
\hline TOTAL & 30 \\
\hline
\end{tabular}

\section{Grafica 3.7. Críticas negativas}

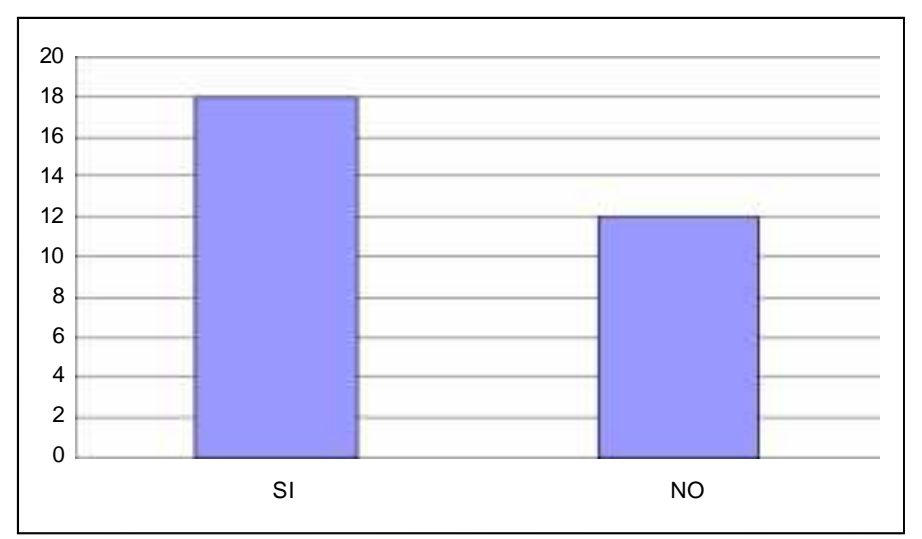

El $60 \%$ de las adolescentes encuestadas son constantemente criticadas de manera negativa por sus parejas y sólo el $40 \%$ de las mismas respondió que lo anterior no les sucede. 
Tabla 1.8. Influencia en la toma de decisiones

\begin{tabular}{|c|c|}
\hline RESPUESTAS & FRECUENCIA \\
\hline SI & 21 \\
\hline NO & 9 \\
\hline TOTAL & 30 \\
\hline
\end{tabular}

\section{Grafica 3.8. Influencia en la toma de decisiones}

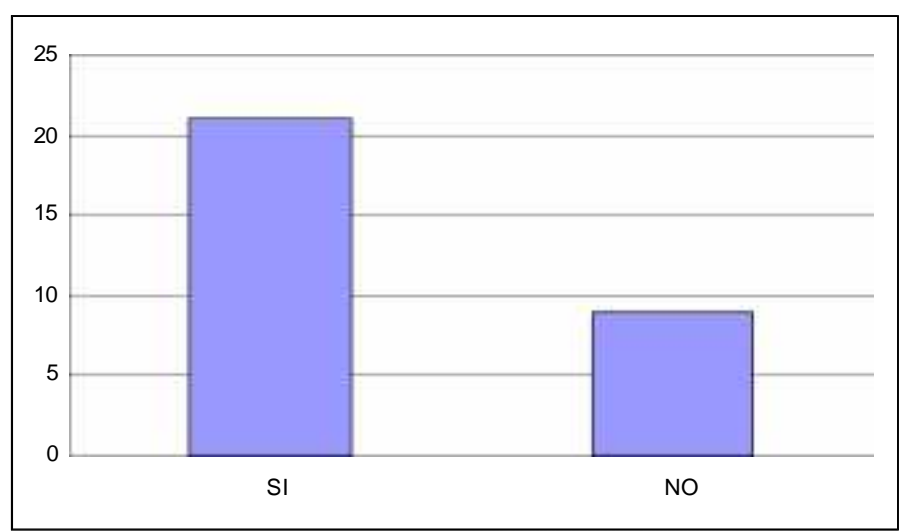

El 70\% de las adolescentes encuestadas son influenciadas por sus parejas en la toma de decisiones, a diferencia del $30 \%$ de ellas que no lo son.

Tabla 1.9. Respeto

\begin{tabular}{|c|c|}
\hline RESPUESTAS & FRECUENCIA \\
\hline SI NO & 7 \\
\hline TOTAL & 23 \\
\hline & 30 \\
\hline
\end{tabular}

\section{Grafica 3.9. Respeto}

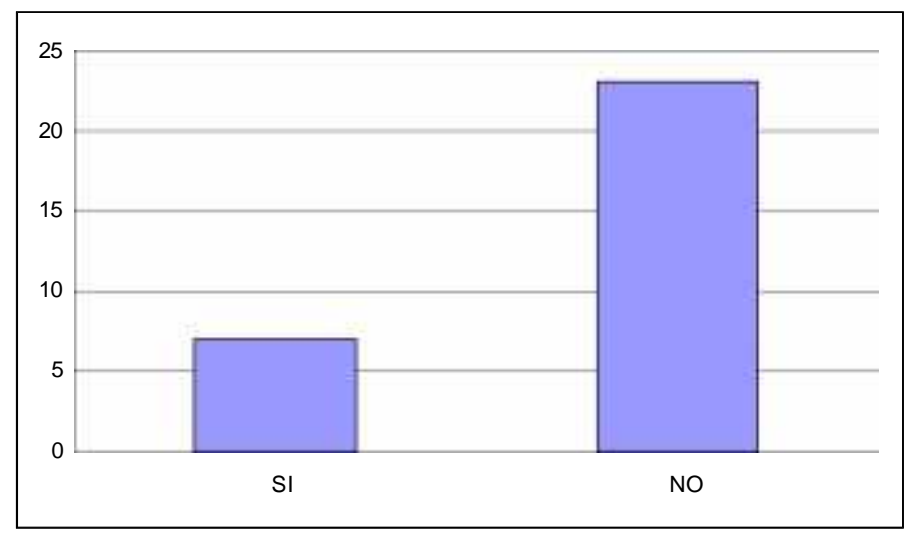

El $76.7 \%$ de las jovencitas sienten que sus parejas no respetan sus hábitos y costumbres, en cambio el $23.3 \%$ de ellas respondieron que sus parejas si lo hacen. 
Tabla 2. Opiniones

\begin{tabular}{|c|c|}
\hline RESPUESTAS & FRECUENCIA \\
\hline SI NO & 16 \\
\hline TOTAL & 14 \\
\hline & 30 \\
\hline
\end{tabular}

\section{Grafica 4. Opiniones}

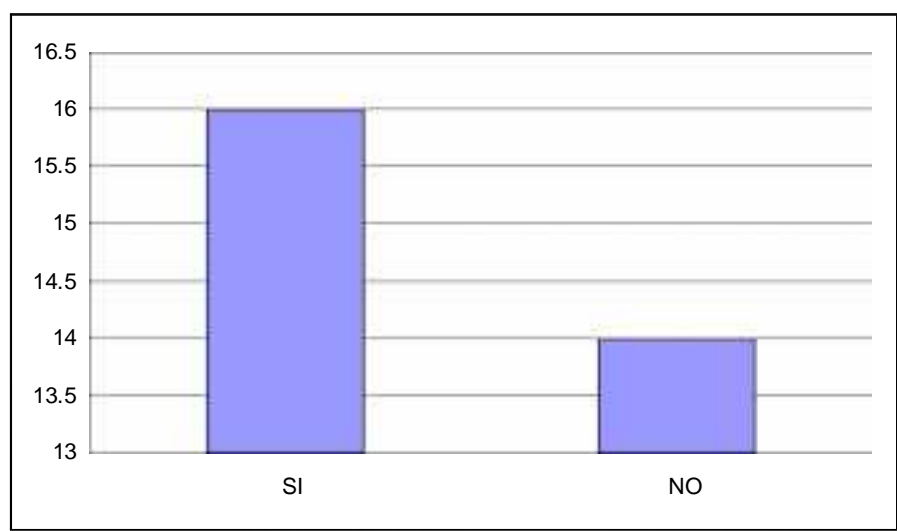

En esta grafica se observa que el 53.3\% de las adolescentes sienten que sus parejas no toman en cuenta sus opiniones, a diferencia del $46.7 \%$ que opinan lo contrario. 


\section{Conclusión}

La violencia se da en algunos noviazgos adolescentes, y puede manifestarse de varias maneras como física, psicológica y/o sexualmente. De acuerdo a los resultados arrojados en esta investigación, actualmente en el noviazgo se presenta mayormente la violencia psicológica; aunque este tipo de violencia no implica heridas físicas, éstas se pueden borrar, en cambio; los recuerdos derivados de la violencia psicológica son mas difíciles de olvidar.

La violencia psicológica en los noviazgos adolescentes actuales es tal, que las jóvenes llegan a cambiar su comportamiento con tal de evitar peleas, al igual que cambian su forma de vestir; además la mayoría de ellas ha perdido contacto con amigos, familiares y compañeros de escuela, con tal de que su pareja no se moleste.

Cabe mencionar que este tipo de relación generalmente se vuelve un tormento para las jóvenes ya que sus parejas se burlan de ellas y las critican constantemente, en pocas palabras son menospreciadas por ellos; y de ser unas jóvenes alegres, seguras de sí mismas, independientes, etc., se pueden transformar en todo lo contrario debido a la presión ejercida por sus novios.

Tomando en cuenta que en la etapa de la adolescencia se hacen presentes las inseguridades y necesidades de aprobación, es común encontrar relaciones donde la mujer deposita su confianza a la pareja esperando recibir aprobación y compañía por parte de él.

Cuando existe un interés y cariño sincero siempre se buscará el bien de la otra persona; el respeto es un valor que debe estar presente en distintos aspectos como lo son las ideas, la toma de decisiones, opiniones, gustos, hábitos costumbres, entre otras cosas. 
De acuerdo con los resultados obtenidos, la mayoría de las jovencitas sienten que sus parejas no respetan sus hábitos ni costumbres, así como tampoco toman en cuenta sus opiniones; es decir, el respeto es un valor que no se encuentra del todo en los noviazgos adolescentes actuales.

Es tan marcada la falta que hace el respeto en las relaciones, que la mayor parte de las adolescentes se ven influenciadas por sus parejas al momento de tomar una decisión; lo que significa perder la individualidad, incluso la fidelidad hacia uno mismo.

Dentro de este escenario es muy fácil encontrar parejas donde la mujer no se da cuenta que no esta recibiendo precisamente lo que espera, y posiblemente este siendo victima de un abuso psicológico, lo que se convierte en un circulo vicioso del que no pueden salir con la esperanza de que la pareja llegará a convertirse en lo anhelado por ser la persona con quien desea estar.

Se considera que esta situación de violencia se hace crítica en la actualidad, pues los adolescentes son los portadores de los cambios culturales, puede llamárseles víctimas del estilo de crianza, del ritmo de vida actual, de las múltiples influencias en los medios que alteran el ideal en educación, o peor aún: víctimas de violencia en el noviazgo. 


\section{Recomendaciones}

Se recomienda a futuros investigadores de violencia ya sea física, sexual o psicológica en el noviazgo, tomen en cuenta dos variables extrañas como lo son las drogas y otros tipos de sustancias. Ya que el consumo de las mismas, puede afectar los resultados de tal manera que una persona que se encuentra bajo la influencia de sustancias como el cristal puede mostrarse agresivo por esa razón. 


\section{Bibliografía}

Hernández Sampieri, "Metodología de la investigación”, editorial McGraw-Hill, 2004.

Krauskopof D., “Adolescencia y Educación”, editorial EUNED, 2000.

Gómez A., “Adolescencia e Identidad”, editorial Trillas, 2001.

Pacay M., "Desarrollo Adolescente”, editorial URANO, 2003.

Aberasturi H., “Adultez Temprana”, UCA Editores, 2000.

Casavantes R., "La sociedad de hoy”, editorial Iberonet, 2000.

Martínez H., "Etapas de la vida", editorial EPOCA, S.A., 2003.

Favieres, "Abusos y consecuencias", ediciones Temas de hoy, 2001.

García P., "La verdad sobre la identidad”, editorial PAIDÓS, 2001.

Moya A.,"Sociedad y Cultura", editorial Grijalbo, 2000.

Barrio, "Identidad de Género", editorial Prentice Hall, 1992.

Troncoso y Tenorio, "Vida y Sociedad", editorial Trillas, 1999.

Suárez V., "Papeles a desarrollar", editorial, Trillas, 2000.

Rivas Z., "Mundo de Valores”, editorial Iberonet, 2000.

Franco E., "Valores y Sociedad", editorial Grijalbo, 2001.

Monge, "La importancia del compromiso" editorial Trillas, 2000.

Baños, "Manifestaciones de la violencia”, editorial PAIDÓS, 2000.

http://www.imjuventud.gob.mx/viogenero_contenido.asp

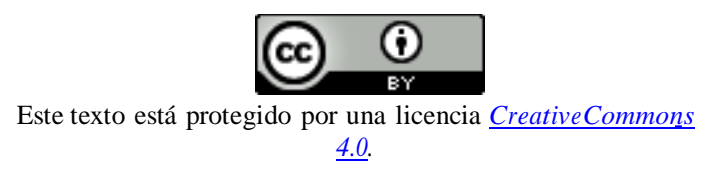

Usted es libre para Compartir — copiar y redistribuir el material en cualquier medio o formato - y Adaptar el documen- to -remezclar, transformar y crear a partir del material- para cualquier propósito, incluso comercialmente, siempre que cumpla la condición de:

Atribución: Usted debe reconocer el crédito de una obra de manera adecuada, proporcionar un enlace a la licencia, e in- dicar si se han realizado cambios. Puede hacerlo en cualquier forma razonable, pero no de forma tal que sugiera que tie- ne el apoyo del licenciante o lo recibe por el uso que hace. 